\title{
Procedure costs associated with the use of Harmonic devices compared to conventional techniques in various surgeries: a systematic review and meta-analysis
}

This article was published in the following Dove Press journal: ClinicoEconomics and Outcomes Research

\author{
Hang Cheng' \\ Jeffrey W Clymer' \\ Rana A Qadeer ${ }^{2}$ \\ Nicole Ferko² \\ Behnam Sadeghirad ${ }^{2}$ \\ Chris G Cameron ${ }^{2}$ \\ Joseph F Amaral' \\ 'Ethicon Inc, Cincinnati, OH, USA; \\ ${ }^{2}$ Cornerstone Research Group, \\ Burlington, ON, Canada
}

Correspondence: Rana A Qadeer Cornerstone Research Group Inc., 204-3228 South Service Road, Burlington, ON L7N 3H8, Canada

Tel + I 905637623 |

Email rqadeer@cornerstone-research. com
Background: As compared to conventional techniques, recent meta-analyses have reported cost savings with Harmonic devices; however, only in thyroidectomy. Thus, the aim of this study was to evaluate the costs associated with Harmonic devices versus conventional techniques across a range of surgical procedures.

Methods: A systematic search of MEDLINE, EMBASE, and Cochrane Library was conducted from inception to October 01, 2016 without language restrictions to identify randomized controlled trials comparing Harmonic devices to conventional techniques and reporting procedure costs (operating time plus operating equipment/consumables/device costs). Costs were pooled using the ratio of geometric means, and a random effects model was applied. Sensitivity analyses varying statistical methods, number of included studies, and cost outcomes were completed to test the robustness of the results.

Results: Thirteen studies met the inclusion criteria. A total of 561 and 540 participants had procedures performed with Harmonic devices and conventional methods, respectively, with procedures including gastrectomy, thyroidectomy, colectomy, cholecystectomy, Nissen fundoplication, and pancreaticoduodenectomy. As compared to conventional methods, Harmonic devices reduced total procedure costs by $8.7 \%(p=0.029)$, resulting in an absolute reduction of US $\$ 227.77$ from mean conventional technique costs, derived primarily from a reduction in operating time costs. When operating time costs, excluding operating equipment/consumables/device costs, were analyzed, costs were reduced by $\$ 544$ per procedure with the use of Harmonic devices. The results from all sensitivity analyses demonstrated cost reductions with Harmonic devices. Conclusion: This systematic review and meta-analysis showed that despite a higher device cost, Harmonic devices provide a statistically significant reduction in procedure costs, derived primarily from a reduction in operating time costs, across surgical procedures. In addition to functionality benefits, Harmonic devices may represent a potentially cost saving method to reduce overall hospital resource use. Future research should focus on potential costs and benefits from use of Harmonic devices in procedures not covered here.

Keywords: Harmonic, ultrasonic, meta-analysis, costs, surgery, efficiency, operating time

\section{Introduction}

Surgical care plays a critical role in preventing, diagnosing, and treating a broad spectrum of diseases across the life span; as such, the global need for surgical procedures is large. In 2010, it was estimated that 321.5 million inpatient surgical procedures were required to address the global burden of disease. ${ }^{1}$ Moreover, researchers have estimated 
that an additional 143 million surgical procedures are needed each year to meet the global need for preventative and curative surgery. ${ }^{2}$ The aging population is expected to further propel the need for surgical care in the foreseeable future. ${ }^{3}$

Given the widespread and indispensable use, ${ }^{2}$ surgical care is associated with substantial costs that have continued to increase unabatedly. In the USA, it is estimated that aggregate surgical expenditures will grow from $\$ 572$ billion in 2005 to $\$ 912$ billion in $2025 .{ }^{4}$ Consequently, payers, hospitals, and health care providers are now placing a greater emphasis on reducing surgical costs to combat the ever-tightening budgetary constraints without sacrificing the quality of care. ${ }^{5}$ Research suggests that one avenue for hospital and surgical cost savings is through the adoption of innovative medical devices and techniques that improve outcomes and reduce surgical complications (eg, bleeding, transfusions). ${ }^{6-8}$

Developed in the early 1990s, Harmonic surgical devices (Harmonic, Ethicon Endo-Surgery, Cincinnati, OH, USA) have emerged as an alternative to conventional hemostasis techniques such as clamp and tie, sutures, metal clips, staples, and monopolar and bipolar electrocautery. Harmonic devices use mechanical vibration to perform simultaneous ultrasonic cutting and coagulation, producing hemostasis at a lower temperature than electrosurgical devices while providing excellent dissecting capability. Meta-analyses have demonstrated that Harmonic devices are associated not only with significant reductions in operating time, ${ }^{9-12}$ blood loss, $,{ }^{9} 10,12,13$ post-operative pain, ${ }^{10}$ complications, ${ }^{13}$ and length of hospital stay, ${ }^{9,13}$ but also with cost savings ${ }^{8,14}$ as compared to conventional methods. In fact, two recent meta-analyses in thyroidectomy have suggested that the use of ultrasonic coagulation (including Harmonic devices) is associated with lower costs as compared to conventional techniques. ${ }^{8,14}$

Presently, meta-analyses examining the costs for Harmonic devices and conventional techniques have not considered surgical procedures other than thyroidectomy. Thus, the aim of this systematic review and meta-analysis of randomized controlled trials (RCTs) was to evaluate the costs associated with Harmonic devices versus conventional techniques across a range of surgical procedures.

\section{Methods}

\section{Literature search}

A systematic search of MEDLINE, EMBASE, and the Cochrane Library was conducted. The search strategy focused on a comprehensive set of terms related to Harmonic devices from inception to October 01, 2016. A detailed search strategy is provided in Figure S1. The reference lists of retrieved articles were also hand-searched.

\section{Study selection}

Specific inclusion criteria were defined according to PICOS (ie, population, intervention, comparator, outcomes, and study design). Studies were considered eligible for inclusion if they were RCTs comparing Harmonic devices to conventional techniques (eg, monopolar or bipolar electrocautery, clips, cut and ligature, knot-tying) in human subjects, for all types of surgical procedures. Based on the inclusion criteria, the eligibility of each publication was evaluated in a title and abstract review. If the abstract and title review suggested potential eligibility, a full-text screening followed. Full-text studies were then excluded if they were not RCTs, did not report cost outcomes, did not provide a cost breakdown (ie, only reported total costs), or did not compare Harmonic devices to conventional techniques. If more than one study using the same dataset met the inclusion criteria, only the most recent or comprehensive (ie, provided the largest amount of data) publication was retained. Records were evaluated for eligibility by two independent reviewers (BS, NF). Disagreements regarding study inclusion were resolved through consensus or consultations with a third reviewer (HC).

\section{Data extraction}

Details (ie, baseline characteristics and outcomes) from the included studies were extracted using a standardized data extraction form developed in Microsoft Excel. The following study details were extracted: study authors, publication year, study time frame, country of origin, sample size, key patient characteristics, type of surgical procedure, type of surgical devices and/or techniques utilized, and detailed cost data. One reviewer conducted the data extraction (BS); a second reviewer cross-checked the extracted data for accuracy and completeness (RAQ). In the case of discrepancies, resolution was reached through consensus.

When available, the type of costs included in the studies were extracted and comprised one or more of the following: operating room (OR) time and/or personnel, OR equipment and/or consumables, preoperative examinations, anesthesia resources, medications, hospital admission/discharge, length of stay, and device-related (eg, Harmonic) costs. Due to inconsistencies in the type and number of cost parameters included across studies, total procedure costs were denoted as the primary outcome of the study. Total procedure costs 
included operating time costs as well as operating equipment/consumables/device costs. Studies $(n=5)$ that reported on costs, but did not provide a breakdown of costs such that procedure-only costs could be extracted, were excluded from the analysis to ensure a consistent definition of costs across all included studies.

\section{Risk of bias}

Risk of bias of individual studies was assessed using the Cochrane Collaboration tool (version 5.1; London, UK). The various domains assessed included sequence generation, allocation concealment, blinding, incomplete outcome data, selective reporting, and other sources of bias. For each domain, the risk of bias was assigned as low, unclear, or high. One author (RAQ) assessed the risk of bias.

\section{Statistical analysis}

For the analysis, mean procedure costs and standard deviations (SDs) in original cost units were extracted from the included studies. All costs were converted to 2016 US dollars (USD) using the Campbell and Cochrane Economics Methods Group and the Evidence for Policy and Practice Information and Coordinating Centre cost converter (Version 1.4) to maximize comparability. If the estimates for the mean and SD were not provided, standard imputation methods outlined by Cochrane were used. ${ }^{15}$ As such, the authors carefully looked for, and used, any statistics that would allow the calculation or estimation of the mean or the SD (eg, range, CIs, standard errors [SEs], $p$-values). For three studies, estimation of the SD was not possible. As per the Cochrane guidelines, SDs were then imputed using the average SD from other studies included in the meta-analysis. Furthermore, the impact of imputation was tested in a sensitivity analysis. ${ }^{15}$

Given that skewness can occur with cost data, the primary meta-analysis compared the procedure costs (2016 USD) for surgical procedures performed with Harmonic devices versus conventional techniques using the ratio of geometric means (RoGM) method. ${ }^{16}$ The natural logarithm and SE of each trial's RoGM were computed using equations presented in Figure S2. The inverse-variance method was then used to aggregate the natural logarithmtransformed ratios across studies in STATA (Version 14.2; StataCorp LP, College Station, TX, USA). A random effects model was used for the meta-analysis and forest plots were generated. Heterogeneity was assessed through the $\chi^{2}$ test. An $I^{2}$ value was also calculated to describe the percentage of variance attributable to heterogeneity among studies.
The following sensitivity analyses were performed to test the robustness of the results: 1) analysis using the ratio of means (RoM) method ${ }^{17}$ instead of the RoGM; 2) a fixed effects model instead of the random effects model; and 3) analysis with the exclusion of imputed studies. An additional sensitivity analysis was completed for operative time costs only (ie, device and consumable costs were excluded) to specifically assess the hospital resource use averted within procedures with the Harmonic device, apart from potentially variable product acquisition costs.

Based on the RoGM or the RoM, percentage reductions in costs were calculated for the primary and sensitivity analyses. These reductions were then converted to absolute reductions by applying the percentage reductions to the mean and range values of the total costs for conventional techniques.

\section{Results Search results}

A total of 2,519 citations were identified. After removing duplicates, 2,125 unique records were reviewed. Following title and abstract review, 1,760 citations were excluded if the studies were not assessing a Harmonic device, not in humans, not comparative, conference abstracts only, grouped Harmonic devices with another device, or conducted in an irrelevant procedure. Of the 365 full texts retrieved for review, 352 were further excluded if the studies were non-RCTs $(n=185)$; did not report cost outcomes $(n=156)$; only included instrument, device, or disposable costs (ie, did not report procedure or operating time costs $)(n=3)$; exclusively reported total costs and did not provide a cost breakdown $(n=5)$; used an irrelevant comparator (ie, LigaSure) $(n=1)$; and were duplicates $(n=2)$. Thirteen studies, consisting of a total of 1,101 patients, that reported costs associated with the use of Harmonic devices and conventional techniques were included in the meta-analysis (Figure 1). ${ }^{18-30}$

The study characteristics are presented in Table 1 . The sample sizes of the included studies ranged from 20 to 261 patients. Six studies reported cost data for thyroidectomy, ${ }^{18,19,22,23,25,26}$ two studies reported data for Nissen fundoplication, ${ }^{24,27}$ two studies reported data for colectomy, ${ }^{20,28}$ and three studies reported data for other surgical procedures (ie, cholecystectomy, ${ }^{29}$ pancreaticoduodenectomy, ${ }^{30}$ and gastrectomy ${ }^{21}$ ). In all studies, Harmonic devices were compared to conventional techniques. The UltraCision Harmonic Scalpel or Shears were used in seven studies, ${ }^{18-20,24,27-29}$ the Harmonic Focus was used in three studies, ${ }^{21,22,26}$ the Harmonic CS-14C ${ }^{25}$ and the Harmonic Wave ${ }^{30}$ were used in one study each, and one study did not specify the type of Harmonic device that was used. ${ }^{23}$ 


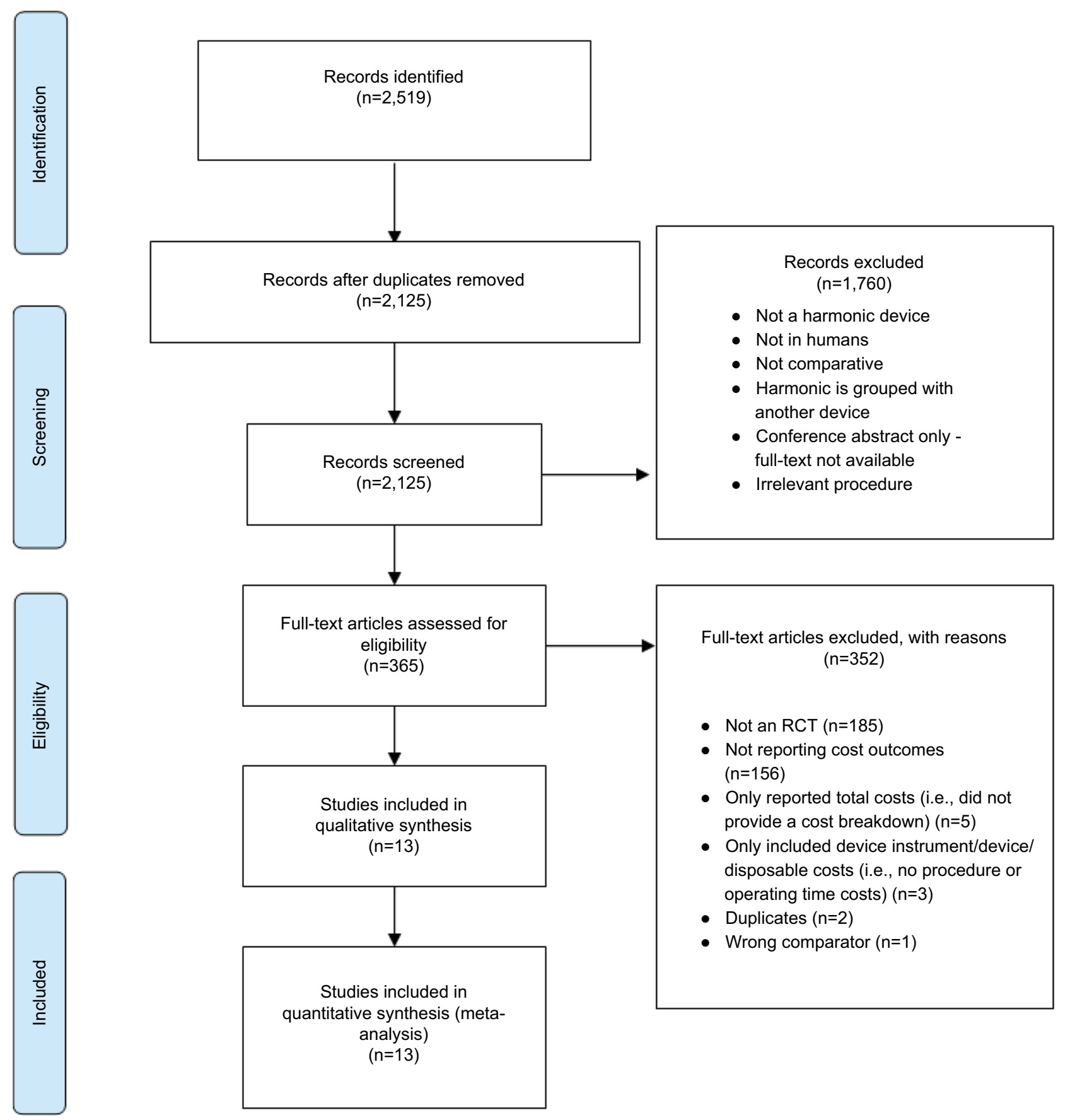

Figure I PRISMA diagram for the systematic literature search.

Abbreviation: $\mathrm{RCT}$, randomized controlled trial.

Different comparators were used for the Harmonic devices in these studies; eight studies used monopolar or bipolar electrocautery with one or more of sutures, clips, clamp and tie, or ligation, ${ }^{19-22,26,28-30}$ while ligatures and cauterization, ${ }^{18}$ cut and ligature, ${ }^{23}$ right-angled multifire clip applier, ${ }^{24}$ knot-tying, ${ }^{25}$ and Ligaclips ${ }^{27}$ were used by one study each. Three studies had three arms (ie, Harmonic device, conventional techniques, LigaSure vessel sealing system $[n=2]$, or electrothermal bipolar vessel sealers $[n=1])$. The third comparator from these studies was excluded from our analyses..$^{20,26,28}$ Most studies ( $\mathrm{n}=9$ ) originated in Europe (eg, Italy, Spain, France, Sweden). Other regions included Japan $(\mathrm{n}=1),{ }^{21}$ Brazil $(\mathrm{n}=1),{ }^{23}$ and USA $(\mathrm{n}=2) \cdot{ }^{24,27}$

\section{Risk of bias assessment}

Risk of bias assessments and individual study quality assessments are presented in Figure S3. The quality of studies varied in terms of random sequence generation, allocation 


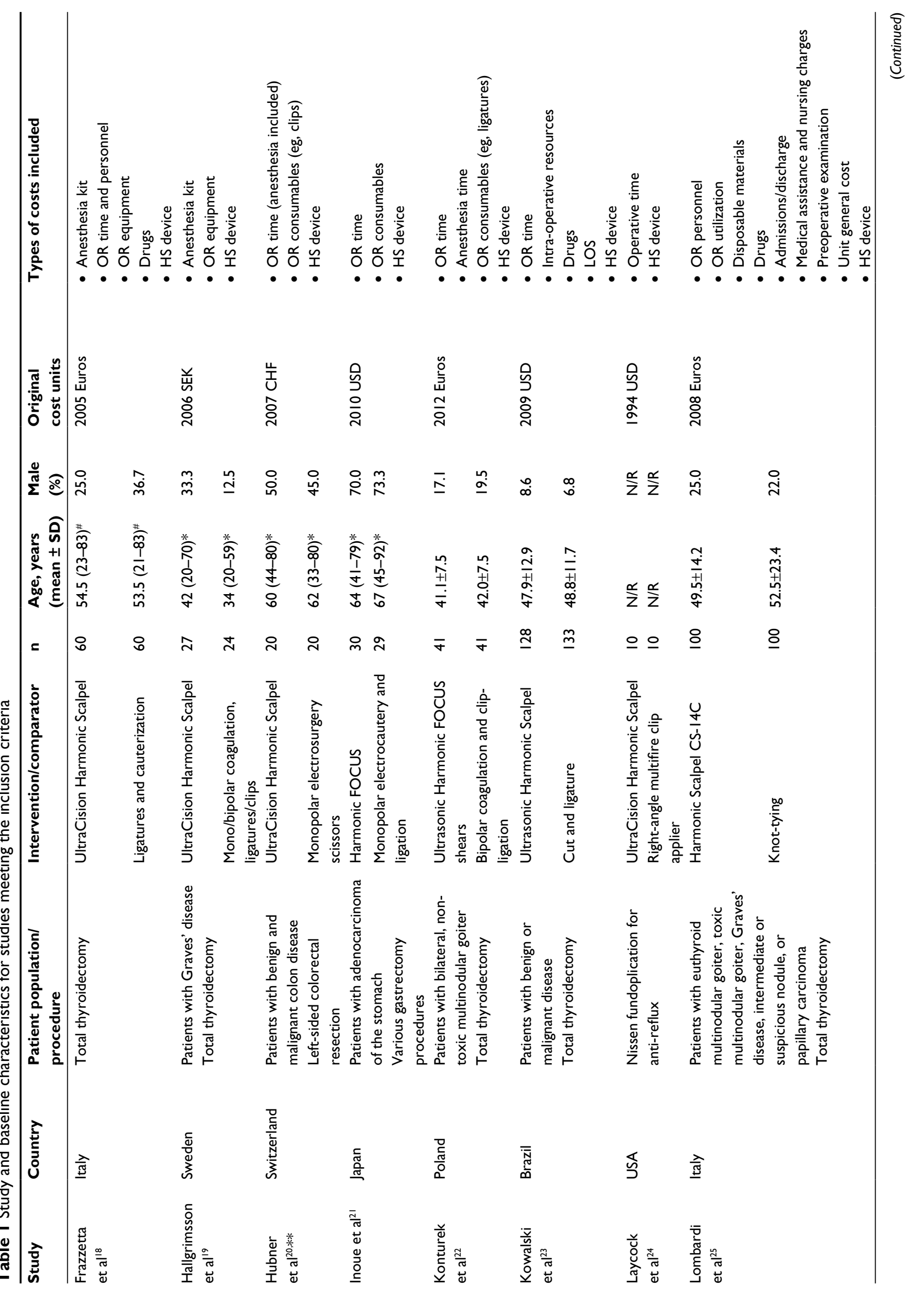




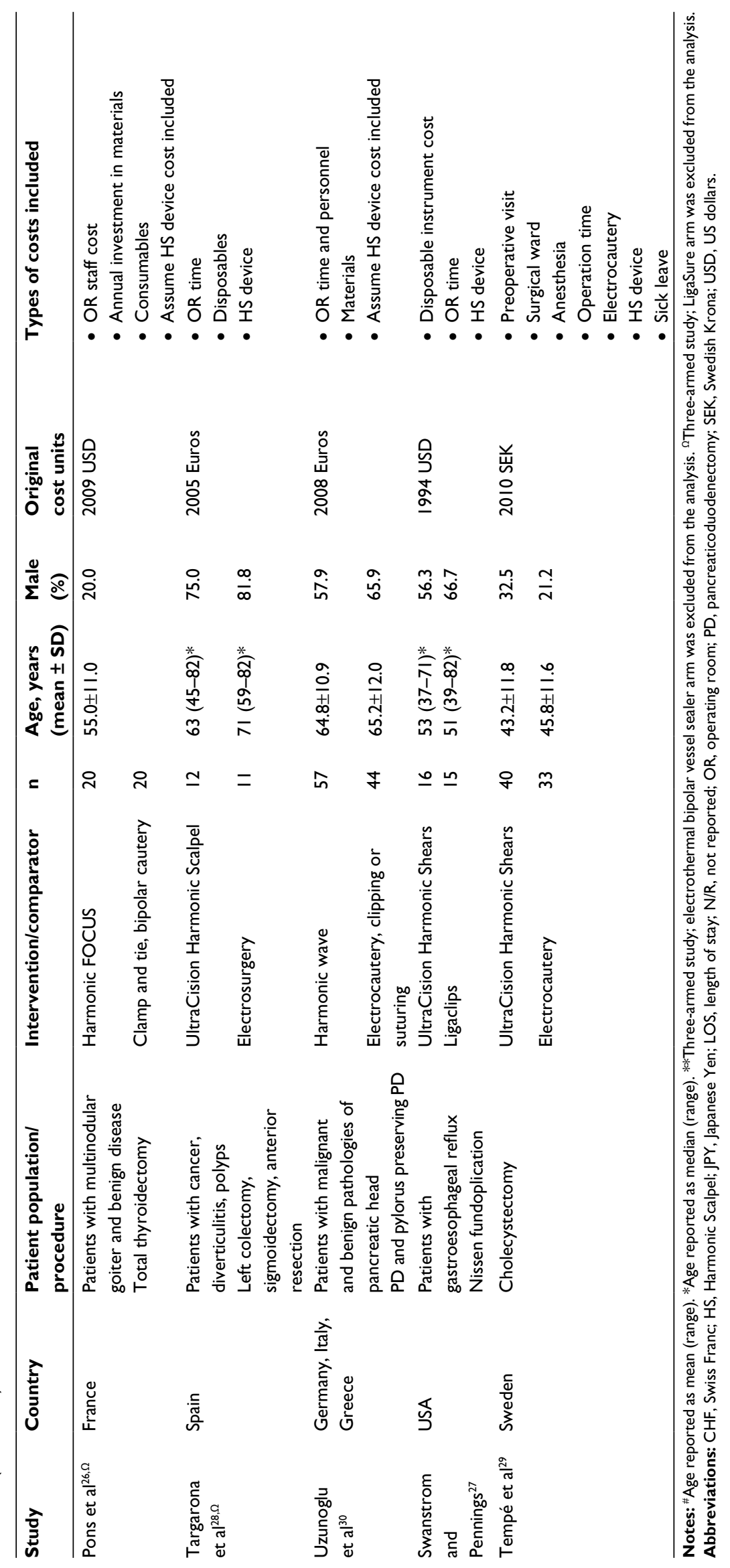


concealment, and selective reporting. Overall, the quality of studies was acceptable, with most studies having low or unclear risk of bias across most domains. Seven studies reported the methods used for random sequence generation such as drawing sealed envelopes $(n=3),{ }^{19,20,22}$ computergenerated random numbers $(\mathrm{n}=3),{ }^{23,28,30}$ and drawing techniques $(n=1) .{ }^{26}$ The methods for allocation concealment were defined by four studies; three studies used sealed envelopes ${ }^{19,20,22}$ and one study used a central telephonic system. ${ }^{23}$ Although six studies reported blinding of patients or outcome assessors, ${ }^{22,23,25,26,29,30}$ the risk of bias was considered low for cost outcomes because of their objectivity (ie, not requiring blinding to avoid bias). Across all studies, patient withdrawals, loss to follow-up, and missing data were minimal. For nine studies, the bias associated with selective reporting was unclear. However, based on protocol assessment, three studies had a low risk of bias ${ }^{21,23,30}$ and one study had a high risk of bias (outcome noted in the methods was not included in the results). ${ }^{26}$

\section{Analysis}

Across all included studies, the mean procedure costs (2016 USD) ranged from $\$ 608.45$ to $\$ 5,290.23$ for surgical procedures conducted with Harmonic devices. Comparatively, the mean procedure costs for surgical procedures performed with the conventional techniques ranged from $\$ 552.87$ to $\$ 5,146.77$ (Table 2). Nine of the 13 included studies (69\%) reported either a statistically significant $(\mathrm{n}=5)$ or numerical $(n=4)$ reduction in procedure costs with the use of Harmonic devices as compared to conventional techniques. The remaining four studies reported effect measures that hovered near cost neutrality, with no studies reporting significantly higher procedure costs with the use of Harmonic devices (Figure 2).

The primary meta-analysis of the 13 included studies showed that procedure costs were statistically significantly reduced by $8.7 \%$ ( $\mathrm{RoGM}=0.913 ; 95 \% \mathrm{CI}=0.842-0.991$; $p=0.029 ; I^{2}=95.8 \%$ ) with the use of Harmonic devices in contrast to conventional techniques (Figures 3 and 4 ). The absolute reduction from mean (conventional) baseline costs

Table 2 Cost inputs and assumptions for primary analysis on procedure costs

\begin{tabular}{|c|c|c|c|c|c|}
\hline \multirow[t]{3}{*}{ Study } & \multirow[t]{3}{*}{ Intervention/comparator } & \multicolumn{4}{|c|}{ Primary analysis } \\
\hline & & \multicolumn{2}{|c|}{$\begin{array}{l}\text { Procedure costs } \\
\text { (original cost units) }\end{array}$} & \multicolumn{2}{|c|}{$\begin{array}{l}\text { Procedure costs } \\
\text { (2016 USD) }\end{array}$} \\
\hline & & Mean & SD & Mean & SD \\
\hline \multirow[t]{2}{*}{ Frazzetta et al ${ }^{18}$} & UltraCision Harmonic Scalpel & $€ 978.60$ & $€ 120.02$ & $\$ 1,517.18$ & $\$ 186.07$ \\
\hline & Ligatures and cauterization & $€ 1,328.68$ & $€ 105.65$ & $\$ 2,059.93$ & $\$ 163.80$ \\
\hline \multirow[t]{2}{*}{ Hallgrimsson et al $^{19}$} & UltraCision Harmonic Scalpel & $18,910.80$ SEK & $N / R$ & $\$ 2,558.56$ & $\$ 552.04 *$ \\
\hline & Mono/bipolar coagulation, ligatures/clips & $22,368.5$ I SEK & $N / R$ & $\$ 3,026.51$ & $\$ 461.14^{\#}$ \\
\hline \multirow[t]{2}{*}{ Hubner et $\mathrm{al}^{20}$} & UltraCision Harmonic Scalpel & I,845.66 CHF & $602.99 \mathrm{CHF}$ & $\$ 1,412.43$ & $\$ 461.45$ \\
\hline & Monopolar electrosurgery scissors & $2,196.58 \mathrm{CHF}$ & $436.35 \mathrm{CHF}$ & $\$ 1,680.98$ & $\$ 333.93$ \\
\hline \multirow[t]{2}{*}{ Inoue et $\mathrm{a}^{21}$} & Harmonic FOCUS & $\$ 4,830.70$ & $\$ 176.17$ & $\$ 5,290.23$ & $\$ 192.93$ \\
\hline & Monopolar electrocautery and ligation & $\$ 4,699.70$ & $\$ 179.18$ & $\$ 5, \mid 46.77$ & $\$ 196.22$ \\
\hline \multirow[t]{2}{*}{ Konturek et $\mathrm{a}^{22}$} & Ultrasonic Harmonic FOCUS shears & $€ 666.20$ & $€ 37.50$ & $\$ 839.87$ & $\$ 47.29$ \\
\hline & Bipolar coagulation and clip-ligation & $€ 718.00$ & $€ 69.20$ & $\$ 905.44$ & $\$ 87.27$ \\
\hline \multirow[t]{2}{*}{ Kowalski et a ${ }^{23}$} & Ultrasonic Harmonic Scalpel & $\$ 2,103.60$ & $\$ 493.70$ & $\$ 2,331.86$ & $\$ 604.18$ \\
\hline & Cut and ligature & $\$ 2,048.40$ & $\$ 846.34$ & $\$ 2,270.23$ & $\$ 938.18$ \\
\hline \multirow[t]{2}{*}{ Laycock et $\mathrm{al}^{24}$} & UltraCision Harmonic Scalpel & $\$ 734.00$ & $\$ 62.30$ & $\$ 1,102.73$ & $\$ 93.60$ \\
\hline & Right-angle multifire clip applier & $\$ 925.00$ & $\$ 185.00$ & $\$ 1,389.68$ & $\$ 277.94$ \\
\hline \multirow[t]{2}{*}{ Lombardi et $\mathrm{al}^{25}$} & Harmonic Scalpel CS-I4C & $€ I, 44 \mid .20$ & $€ 260.22$ & $\$ 2,088.79$ & $\$ 377.15$ \\
\hline & Knot-tying & $€ I, 600.70$ & $€ 289.57$ & $\$ 2,319.97$ & $\$ 419.69$ \\
\hline \multirow[t]{2}{*}{ Pons et $\mathrm{al}^{26}$} & Harmonic FOCUS & $€ 2,486.00$ & $€ 153.00$ & $\$ 2,755.76$ & $\$ 169.60$ \\
\hline & Clamp and tie, bipolar cautery & $€ 2,571.00$ & $€ 296.00$ & $\$ 2,849.98$ & $\$ 328.12$ \\
\hline Swanstrom and & UltraCision Harmonic Shears & $\$ 405.00$ & $N / R$ & $\$ 608.45$ & $\$ 552.04 *$ \\
\hline Pennings ${ }^{27}$ & Ligaclips & $\$ 368.00$ & $N / R$ & $\$ 552.87$ & $\$ 461.14^{\#}$ \\
\hline \multirow[t]{2}{*}{ Targarona et a $\left.\right|^{28}$} & UltraCision Harmonic Scalpel & $€ 2,928.00$ & $€ 895.66$ & $\$ 4,909.8 \mathrm{I}$ & $\$ 1,501.89$ \\
\hline & Electrosurgery & $€ 2,995.00$ & $€ 321.68$ & $\$ 5,022.16$ & $\$ 539.41$ \\
\hline \multirow[t]{2}{*}{ Tempé et a $\left.\right|^{29}$} & UltraCision Harmonic Shears & I5,650.00 SEK & $N / R$ & $\$ 1,926.48$ & $\$ 552.04 *$ \\
\hline & Electrocautery & $16,840.00$ SEK & $N / R$ & $\$ 2,072.96$ & $\$ 461.14^{\#}$ \\
\hline \multirow[t]{2}{*}{ Uzunoglu et $\mathrm{al}^{30}$} & Harmonic wave & $€ 3,630.00$ & $€ I, 354.08$ & $\$ 5,056.50$ & $\$ 1,886.20$ \\
\hline & Electrocautery, clipping or suturing & $€ 3,401.00$ & $€ 952.55$ & $\$ 4,737.51$ & $\$ 1,326.88$ \\
\hline
\end{tabular}

Notes: *Imputed as the average of the reported SDs from other studies included in the meta-analysis assessing the costs of Harmonic devices. \#Imputed as the average of the reported SDs from other studies included in the meta-analysis assessing the costs of conventional techniques.

Abbreviations: CHF, Swiss Franc; JPY, Japanese Yen; N/R, not reported; SEK, Swedish Krona; USD, US dollars. 


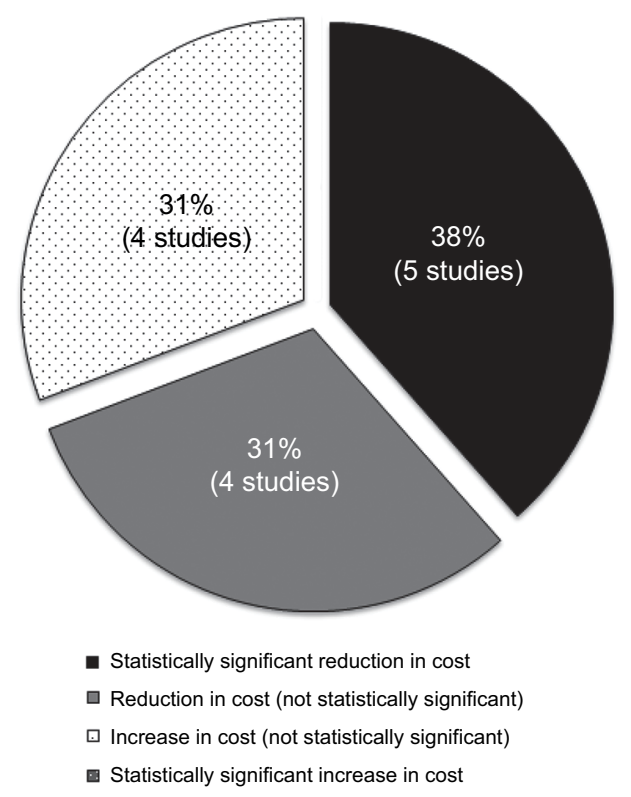

Figure 2 Proportion of studies reporting statistically significant or nonsignificant reductions or increases in procedure costs using the Harmonic devices as compared to conventional methods. was then calculated to be $\$ 227.77$ (range $=\$ 48.10-\$ 447.77$ ) (Table 3). The results from all sensitivity analyses demonstrated cost reductions with the use of Harmonic devices as compared to conventional techniques (Table 3; Figure 5). When the RoM method was used, a statistically significant reduction of $8.3 \%$ (RoM $=0.917 ; 95 \% \mathrm{CI}=0.847-0.994$; $\left.p=0.034 ; I^{2}=95.6 \%\right)$ in mean procedure costs or an absolute reduction of $\$ 217.30$ (range $=\$ 45.89-\$ 427.18$ ) was noted with the use of Harmonic devices (Figure S4). Similar results were observed when operative time costs, reported by 11 of the 13 included studies, were analyzed. When these studies were meta-analyzed, a statistically significant reduction of $30.6 \%$ or $29.1 \%$ was reported by the RoGM $\left(0.694 ; 95 \% \mathrm{CI}=0.603-0.799 ; p<0.001 ; I^{2}=95.6 \%\right)$ and the $\operatorname{RoM}\left(0.709 ; 95 \%\right.$ CI $\left.=0.621-0.810 ; p<0.001 ; I^{2}=95.1 \%\right)$ methods in favor of the Harmonic devices, respectively (Table 3, Figure 4, Figures S5 and S6). Consequently, the Harmonic devices resulted in an absolute reduction of $\$ 544.45$ (range $=\$ 101.14-\$ 1,552.22)$ or $\$ 517.76$

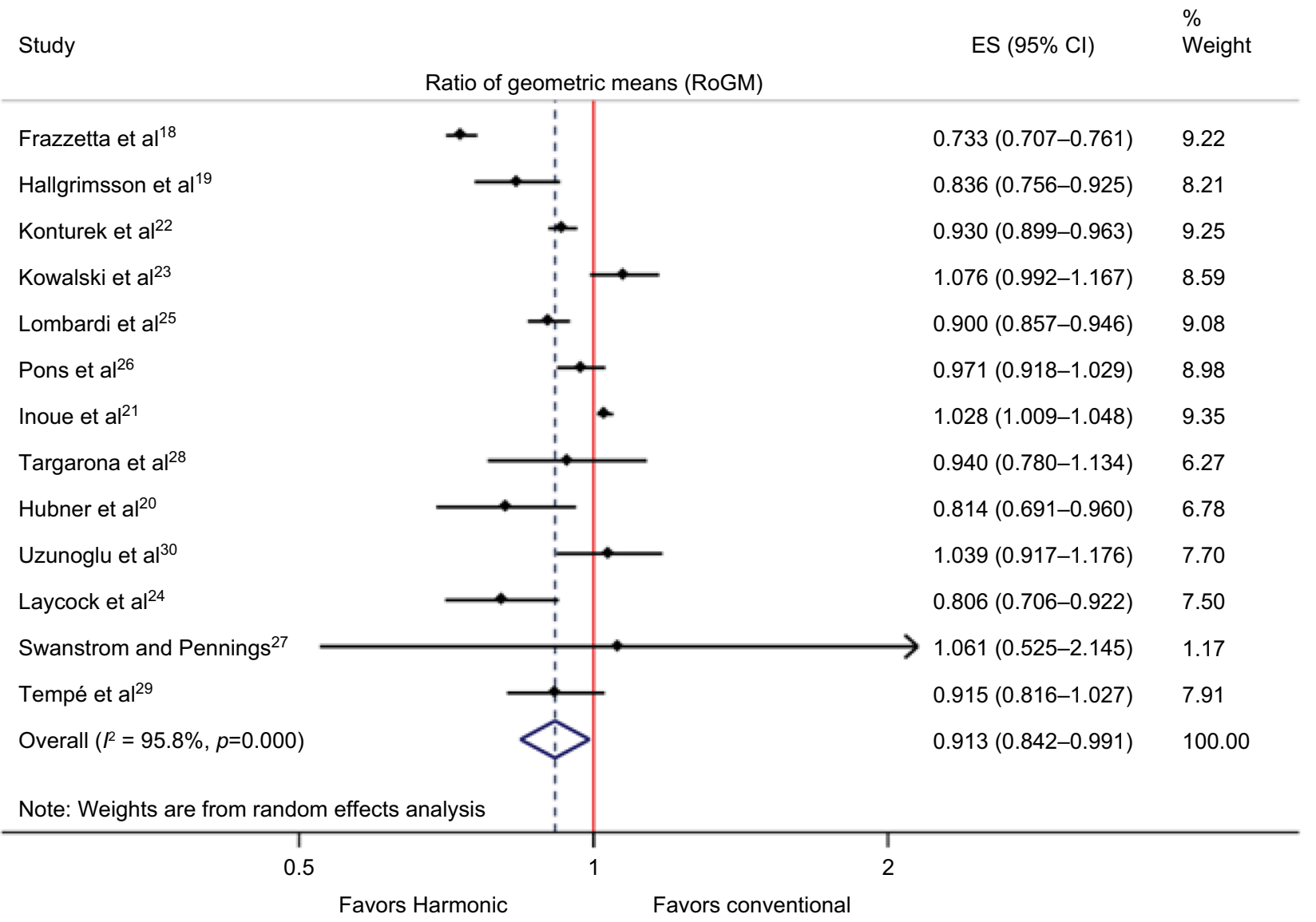

Figure 3 Forest plot of primary meta-analysis results for procedure costs (2016 US dollars [USD]) expressed as ratio of geometric means (RoGM); $p$-value (test of effect size $[E S]=1)=0.029$. 
(range $=\$ 96.18-\$ 1,476.14)$ in mean (conventional) baseline costs if only operative time costs were considered (ie, initial acquisition costs of the Harmonic devices and consumables were excluded) using the RoGM and the RoM

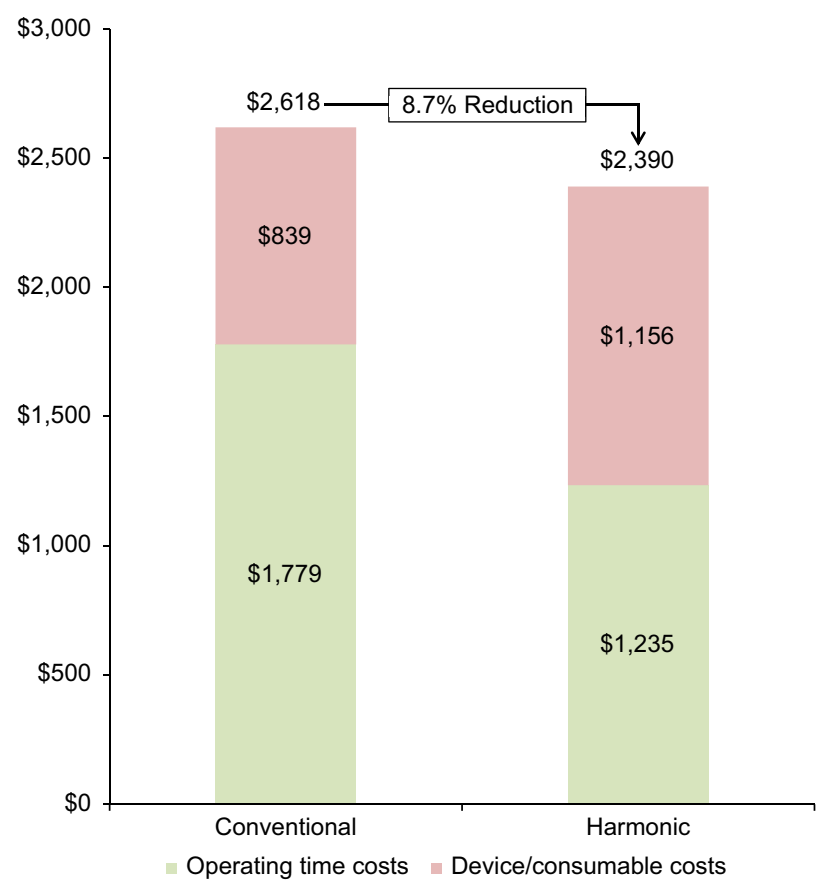

Figure 4 Cost breakdown by operating time costs and device/consumable (ie, anesthesia, clips, ligature, gauze, drugs, intraoperative resources) costs for Harmonic and conventional techniques using the ratio of geometric means (RoGM) method. Although the device/consumable cost for Harmonic is higher, the lower operating time costs offset these higher costs, such that the total procedure costs for Harmonic are $8.7 \%$ lower than those for conventional techniques $(p=0.029)$. methods, respectively. However, as might be expected, the device/consumable costs were 38\% higher in the Harmonic group (Figure 4). As mentioned earlier, the SDs had to be imputed for three studies; ${ }^{19,27,29}$ when these studies were excluded from the analysis, Harmonic devices were associated with a numerical reduction of $8.1 \%$ in procedure costs (RoGM=0.919; 95\% CI=0.839-1.007; $p=0.071 ; I^{2}=96.8 \%$ ) (Figure S7). Finally, if a fixed-effects model was used, a statistically significant reduction of 5.2\% (RoGM=0.948; $95 \% \mathrm{CI}=0.935-0.961, p<0.001 ; I^{2}=95.8 \%$ ) or an absolute reduction of $\$ 136.14$ (range $=\$ 28.75-\$ 267.63$ ) was noted (Figure S8).

\section{Discussion}

Surgical costs are rising and one method to help control such costs is by using innovative and effective technologies that reduce hospital resource use while improving patient outcomes. Harmonic devices have demonstrated effectiveness and safety across a wide variety of surgical procedures. Using the most comprehensive evidence-based available and robust statistical methods, our findings suggest that Harmonic devices can provide cost savings to hospital payers across many types of surgeries. Specifically, this meta-analysis of 13 studies showed that Harmonic devices were associated with a statistically significant reduction in total costs of $\sim \$ 228$ USD per procedure compared with conventional methods. These findings remained consistently in favor of Harmonic devices when a range of sensitivity analyses were conducted. Based on the methods

Table 3 Percentage and absolute reduction in procedure costs with Harmonic devices compared with conventional techniques

\begin{tabular}{|c|c|c|c|c|c|}
\hline & $\begin{array}{l}\text { Studies } \\
\text { informing } \\
\text { the analysis }\end{array}$ & $\begin{array}{l}\text { Effect estimate } \\
(95 \% \mathrm{Cl}) ; p \text {-value* }\end{array}$ & $\begin{array}{l}\text { Reduction } \\
\text { in cost } \\
(\%)\end{array}$ & $\begin{array}{l}\text { Mean baseline } \\
\text { procedure costs in } 2016 \\
\text { USD (conventional } \\
\text { technique) }\end{array}$ & $\begin{array}{l}\text { Absolute reduction } \\
\text { in cost in } 2016 \text { USD, } \\
\text { mean (range) }\end{array}$ \\
\hline $\begin{array}{l}\text { Total procedure costs } \\
\text { (RoGM) }\end{array}$ & 13 & $\begin{array}{l}0.913(0.842-0.991) \\
p=0.029\end{array}$ & $8.7 \%$ & $\$ 2,618.08$ & $\$ 227.77$ (48.10-447.77) \\
\hline \multicolumn{6}{|l|}{ Sensitivity analyses: } \\
\hline Total procedure costs (RoM) & 13 & $\begin{array}{l}0.917(0.847-0.994) \\
p=0.034\end{array}$ & $8.3 \%$ & $\$ 2,618.08$ & $\$ 217.30(45.89-427.18)$ \\
\hline $\begin{array}{l}\text { Operating time costs only } \\
\text { (RoGM) }^{\infty}\end{array}$ & 11 & $\begin{array}{l}0.694(0.603-0.799) \\
p<0.001\end{array}$ & $30.6 \%$ & $\$ 1,779.25$ & $\$ 544.45$ (101.14-1552.22) \\
\hline Operating time costs only (RoM) ${ }^{\infty}$ & 11 & $\begin{array}{l}0.709(0.62 \mathrm{I}-0.8 \mathrm{I} 0) \\
p<0.00 \mathrm{I}\end{array}$ & $29.1 \%$ & $\$ 1,779.25$ & $\$ 517.76(96.18-1476.14)$ \\
\hline $\begin{array}{l}\text { Total procedure costs (no } \\
\text { imputation) (RoGM)** }\end{array}$ & 10 & $\begin{array}{l}0.919(0.839-1.007) \\
p=0.07 \mid\end{array}$ & $8.1 \%$ & $\$ 2,838.27$ & $\$ 229.90(73.34-416.89)$ \\
\hline $\begin{array}{l}\text { Total procedure costs using a fixed } \\
\text { effects model (RoGM) }\end{array}$ & 13 & $\begin{array}{l}0.948(0.935-0.961) \\
p<0.001\end{array}$ & $5.2 \%$ & $\$ 2,618.08$ & $\$ 136.14(28.75-267.63)$ \\
\hline
\end{tabular}

Notes: ${ }^{*} \mathrm{~A}$ random effects model was applied if not specified. ${ }^{\circ}$ Excludes Konturek et al, ${ }^{22}$ and Uzunoglu et al. ${ }^{30}{ }^{* *}$ Excludes Hallgrimsson et al, ${ }^{19}$ Swanstrom and Pennings, ${ }^{27}$ and Tempé et al. ${ }^{29}$

Abbreviations: RoM, ratio of means; RoGM, ratio of geometric means; USD, US dollars. 


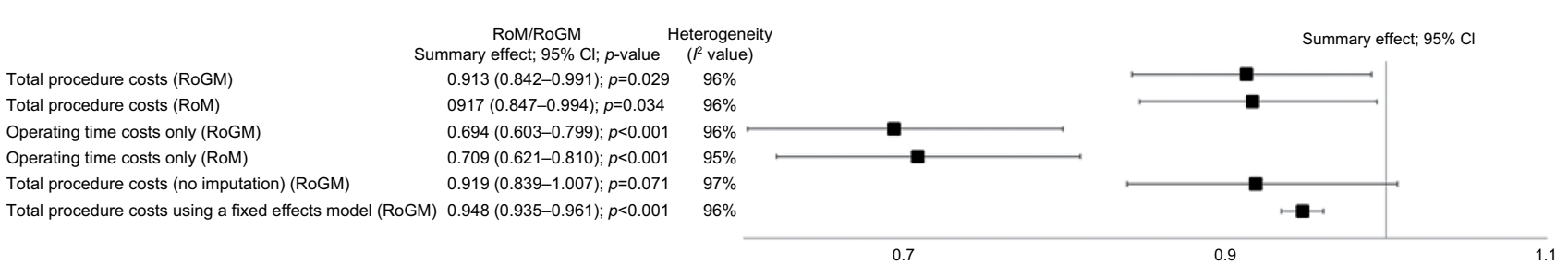

Figure 5 Forest plot of ratio of geometric means (RoGM) and ratio of means (RoM) summary effect measures for the primary and sensitivity analyses.

outlined by the Cochrane Collaboration, most included studies were of acceptable quality with low risk of bias.

The studies within our meta-analysis included a range of surgical procedures, many of which are commonly performed and thus are of high relevance. In a recent health care cost and utilization project (HCUP) statistical report, two of the procedure types included (ie, cholecystectomy and colorectal resection) were shown to be amongst the 15 most common inpatient procedures completed in the USA in 2012. ${ }^{31}$ Furthermore, gastrectomy, which was a focus of one study in our meta-analysis, is a procedure that showed the greatest rate of change over the period of 2003-2012 of all procedures assessed in the HCUP report, increasing in frequency by $150 \%$ from 9.3 to 23.6 per 100,000 individuals. ${ }^{31}$ Thyroidectomy, a focus of several of the studies in our meta-analysis, is also a common procedure, with over 130,000 procedures completed each year in the USA. ${ }^{32}$

The results presented in the current meta-analysis corroborate findings from studies that have compared cost outcomes between ultrasonic devices (including Harmonic) and conventional methods. All published meta-analyses to date have demonstrated either numerical or statistically significant cost reductions with ultrasonic/Harmonic devices; however, these studies focused solely on thyroid surgery. ${ }^{8,14}$ With the most recently published meta-analysis, Cheng et al, ${ }^{8}$ demonstrated that Harmonic devices were associated with a statistically significant reduction of $10 \%$ or $\$ 229$ USD in total reported costs as compared with conventional techniques based on seven studies in thyroidectomy. The current meta-analysis, including a greater number of studies as well as additional procedure types, demonstrated findings that were aligned with the recent Cheng et al's publication, showing a statistically significant reduction of $8.7 \%$ or $\$ 228$ USD in procedure costs with Harmonic devices $($ RoGM $=0.913 ; 95 \% \mathrm{CI}=0.842-0.991 ; p=0.029 ; 13$ studies). Furthermore, the methods of this latter published meta-analysis are highly aligned to those of the current study as they adhered to best practices and methodological rigor to address pooling of cost data across regions. ${ }^{8}$ In brief, these methods included the conversion of baseline costs to a common currency (ie, USD), inflation of costs to a common year (eg, 2016), and use of the RoGM and RoM effect measures to handle skewness that can occur with cost data and ease interpretation of outcomes expressed in different units.

As surgical needs continue to grow, increasing economic burden is inevitable. With rising costs, it is essential that decision-making for the adoption of new surgical technologies comprehensively considers clinical, efficiency, and economic outcomes. As such, there is a greater push towards use of increasingly efficient and cost-effective technologies. ${ }^{33}$ Defining cost-effectiveness in the context of surgical devices in the hospital setting can be variable. Nevertheless, an innovative device with an acquisition cost that is higher than current care should improve outcomes and thereby help reduce downstream health care resource use. Such resources could include both direct and indirect costs such as operating staff time, nursing time, hospital days, complication treatments, re-operations, and additional product use. From a hospital purchaser's perspective, a technology that is cost-effective could be one where costs (including upfront and downstream) are higher for the new technology but the additional benefit achieved is deemed to be worth the extra cost. A cost neutral technology could be one whereby savings in downstream resources completely offset its additional upfront cost, which is still a very desirable situation given that there is no increase in cost but there is additional benefit. A cost saving technology offers incremental clinical benefit and is associated with total costs that are less than current standard of care. The results of our analysis fit into this latter category; Harmonic devices were associated with a statistically significant reduction in procedure costs compared with conventional methods. When assessing individual studies that comprised the meta-analysis, studies that did not show a reduction in procedure costs still showed results that were cost neutral for the Harmonic devices and no studies reported statistically significantly higher procedure costs with Harmonic 
devices as compared to conventional methods (still a desirable scenario).

Our analysis demonstrated that a large part of the cost savings associated with the Harmonic devices was due to reduced operating time costs (excluding the consumable cost of Harmonic) (Figure 5). As compared to conventional techniques, Harmonic devices incorporate multiple functions into one device (ie, perform simultaneous cutting and coagulation) and lead to improvements in both the control of the hemostasis and the precision of tissue dissection, thereby minimizing operating time. ${ }^{34}$ Several studies have demonstrated reduced operative time with Harmonic devices as compared to conventional techniques in different surgical areas, including gastrectomy, ${ }^{12}$ mastectomy, ${ }^{13}$ thyroidectomy, ${ }^{35}$ cholecystectomy, ${ }^{36}$ and parotidectomy. ${ }^{37}$ Previous meta-analyses of the Harmonic devices have shown that operating time can be reduced by up to 30 minutes. ${ }^{12,35}$ This can reduce resource use such as operative staff time, anesthesia time, and operative equipment and materials. Another important concept that was not factored into this analysis is that of opportunity cost. Chatterjee et $\mathrm{a}^{33}$ discussed the notion of surgical opportunity cost and built a model to estimate this for various surgeries by considering procedure volume, profit margin, and surgery duration. In essence, if operating time is extended, this reduces the potential for fitting in more surgeries and obtaining further profits from those surgeries. For example, the opportunity cost per minute of general surgery was predicted to be $\$ 9$ per minute, with higher procedure profit margins leading to even higher opportunity cost multipliers. ${ }^{33}$ If a less efficient technology added 30 minutes of operating time relative to a more efficient comparator, it would be associated with an opportunity cost of $\$ 270$. This concept becomes more applicable for more common procedures as it would be easier to accumulate a sufficient number of cases to accrue the time necessary to perform an additional profitable procedure. As such, even if the fixed and direct costs of a device are high, the device can be extremely desirable if the surgical procedure has a high opportunity cost per minute and the device substantially reduces operating time. ${ }^{33}$

When the costs of the Harmonic devices and other consumables were excluded, a reduction of $30.6 \%$ or $\$ 544$ per procedure was noted in our meta-analysis. Of course, there will always be an acquisition cost for the Harmonic device; however, it is likely to vary across regions and hospitals. Analyses that exclude the acquisition cost may allow regional consideration of the results in context of local Harmonic acquisition prices. Clearly, the lower the Harmonic device cost, the higher the potential for greater cost savings to be achieved. The cost per minute of operating time can also vary across regions. In a few of the European studies included in our analysis, a low cost of $\sim € 4$ per minute was integrated into the study's analysis. ${ }^{19,22}$ In the USA, higher operating costs per minute have been reported in recent literature, for example, a value of $\$ 62$ per OR minute has been quoted in the USA. ${ }^{38}$ In general, reduction of operating time can provide substantial benefit for surgical practice; however, the saving of operating time should be set in relation to higher material costs, which needs to be evaluated within each institution. ${ }^{39}$

This systematic review and meta-analysis has several strengths including the comprehensive literature search, inclusion of a greater number of studies than any other previously published cost meta-analysis assessing Harmonic devices, assessment of study quality, and the use of the most appropriate statistical methods for pooling and interpreting cost data. Furthermore, this meta-analysis included studies from various regions (ie, North America, Europe, Australia, South America, Asia) across the world. The findings, however, should be interpreted in the context of the following limitations. First, the meta-analyses yielded high heterogeneity, which may have been due to factors such as pooling cost data across surgical procedures, smaller number of studies within a specific surgical procedure (eg, only one study assessed cost outcomes in gastrectomy), and variability in study populations and settings. As such, a random effects model, which accommodates for heterogeneity, was used. Moreover, multiple sensitivity analyses demonstrated that the results remained robust and supported the main conclusions. As further data become available for cost outcomes with the Harmonic device across the procedure types studied, subgroup analyses on various procedure types may be more achievable. Future research should also focus on the cost-effectiveness of Harmonic devices in procedures not covered here and as compared to other newer techniques and devices. Until such analyses are completed, the authors note that the results from this analysis are restricted to the surgical procedures included in the presented analyses (ie, gastrectomy, thyroidectomy, colectomy, cholecystectomy, Nissen Fundoplication, and pancreaticoduodenectomy). Second, a few studies that assessed cost outcomes for the Harmonic device could not be included because procedure costs were not reported (ie, studies only reported device or disposable costs or exclusively reported total costs without providing a cost breakdown). In brief, these studies reported mixed findings on whether the Harmonic scalpel saved or 
added costs in procedures such as cholecystectomy, Nissen fundoplication, breast surgery, hysterectomy, gastrectomy, and thyroidectomy; ${ }^{40-47}$ however, it would be impossible to predict how these findings could have impacted the current meta-analysis in the absence of procedure costs. Third, not all studies specified the individual cost components that were present in their cost analyses. Where possible, these details were extracted and reported in the results of this manuscript. Although it is reasonable to assume that many of the core variables such as operating time, surgical materials, and devices costs were captured in all study-specific analyses, some inconsistencies such as the use of charges versus costs existed. Future costing studies in this area should clearly delineate all cost variables as well as capture total hospital-related costs (including procedure costs) where possible to optimize accuracy and utility of cost analyses for decision-making. Fourth, conference abstracts, which might have relevant cost information, especially for recent studies without a manuscript, were excluded, potentially increasing the risk of publication bias. However, published literature has suggested that conference abstracts frequently report preliminary results, which may not be representative of the final results. ${ }^{48}$ Furthermore, conference abstracts often involve insufficient information to determine study validity, a lack of a rigorous peer review process, and uncertainty regarding the methodological quality of evidence. ${ }^{49}$ With our analysis, it was also essential to understand the individual components included within the total reported costs, which are often only detailed in full-text publications. Fifth, the results of this analysis may not be entirely generalizable across settings, as resource use and costs are variable both within and between countries. Although several regions were included in the analysis, local variation in the key components of this study, namely Harmonic device prices and cost per OR minute, needs to be considered. Our analysis can nevertheless be used as a framework for guiding institutionspecific assessments.

\section{Conclusion}

This systematic review and meta-analysis provided a cost comparison of Harmonic devices with conventional techniques in various surgical procedures. The results showed a statistically significant reduction of $8.7 \%$ in procedure costs or $\sim \$ 228$ USD per procedure in favor of the Harmonic devices. Therefore, Harmonic devices may represent a potentially cost saving hemostatic method for various surgical procedures, as reductions in hospital resource use (eg, operative time) may offset the device costs. Future research should focus on the cost-effectiveness of Harmonic devices in procedures not covered here and as compared to other newer techniques and devices.

\section{Acknowledgments}

This work was sponsored by Ethicon, Inc., manufacturer of the Harmonic devices, who provided funding to conduct the analysis and prepare the manuscript.

\section{Author contributions}

$\mathrm{HC}$, JWC, and JFA were involved in the conception and design of the study, interpretation of the data, revising paper critically for intellectual content, and final approval. RAQ, $\mathrm{NF}$, and BS were involved in data acquisition, data analysis, interpretation of the data, drafting of the paper, and final approval. CGC was involved in analyses and interpretation of the data, revising the paper critically for intellectual content, and final approval. All the authors agree to be accountable for all aspects of the work.

\section{Disclosure}

$\mathrm{HC}$, JWC, and JFA are employees of Ethicon, Inc., manufacturer of the Harmonic devices. RAQ, NF, BS and CGC are employees of Cornerstone Research Group, who were sponsored to perform this study by Ethicon, Inc. The authors report no other conflicts of interest in this work.

\section{References}

1. Rose J, Weiser TG, Hider P, Wilson L, Gruen RL, Bickler SW. Estimated need for surgery worldwide based on prevalence of diseases: a modelling strategy for the WHO Global Health Estimate. Lancet Glob Health. 2015;3 Suppl 2:S13-S20.

2. Meara JG, Leather AJ, Hagander L, et al. Global Surgery 2030: evidence and solutions for achieving health, welfare, and economic development. Lancet. 2015;386(9993):569-624.

3. Etzioni DA, Liu JH, Maggard MA, Ko CY. The aging population and its impact on the surgery workforce. Ann Surg. 2003;238(2):170-177.

4. Muñoz E, Muñoz W 3rd, Wise L. National and surgical health care expenditures, 2005-2025. Ann Surg. 2010;251(2):195-200.

5. Ismail I, Wolff S, Gronfier A, Mutter D, Swanström LL. A cost evaluation methodology for surgical technologies. Surg Endosc. 2015;29(8):2423-2432.

6. Snowdon A, Zur R, Shell J. Transforming Canada into a Global Centre for Medical Device Innovation and Adoption. London, ON: Ivey Centre for Health Innovation and Leadership, University of Western Ontario; 2011.

7. Vonlanthen R, Slankamenac K, Breitenstein S, et al. The impact of complications on costs of major surgical procedures: a cost analysis of 1200 patients. Ann Surg. 2011;254(6):907-913.

8. Cheng H, Soleas IM, Ferko NC, Cameron CG, Clymer JW, Amaral JF. Hospital costs associated with thyroidectomy performed with a Harmonic device compared to conventional techniques: a systematic review and meta-analysis. J Med Econ. 2016;19(8):750-758.

9. Cannizzaro MA, Borzì L, Lo Bianco S, Okatyeva V, Cavallaro A, Buffone A. Comparison between Focus Harmonic scalpel and other hemostatic techniques in open thyroidectomy: a systematic review and meta-analysis. Head Neck. 2016;38(10):1571-1578. 
10. Revelli L, Damiani G, Bianchi CB, et al. Complications in thyroid surgery. Harmonic Scalpel, Harmonic Focus versus Conventional Hemostasis: a meta-analysis. Int J Surg. 2016;28 Suppl 1:S22-S32.

11. Ren ZH, Xu JL, Fan TF, Ji T, Wu HJ, Zhang CP. The harmonic scalpel versus conventional hemostasis for neck dissection: a meta-analysis of the randomized controlled trials. PLoS One. 2015;10(7):e0132476.

12. Cheng H, Hsiao CW, Clymer JW, et al. Gastrectomy and D2 lymphadenectomy for gastric cancer: a meta-analysis comparing the harmonic scalpel to conventional techniques. Int J Surg Oncol. 2015;2015:397260.

13. Cheng H, Clymer JW, Ferko NC, et al. A systematic review and meta-analysis of Harmonic technology compared with conventional techniques in mastectomy and breast-conserving surgery with lymphadenectomy for breast cancer. Breast Cancer (Dove Med Press). 2016;8:125-140.

14. Zhang ZJ, Zhang P, Tian JH, et al. Ultrasonic coagulator for thyroidectomy: a systematic review of randomized controlled trials. Surg Innov. 2010;17(1):41-47.

15. Higgins JPT, Green S. The Cochrane Handbook for Systematic Reviews of Interventions. Oxford: Cochrane Collaboration; 2011. Available from: http://handbook-5-1.cochrane.org/.

16. Friedrich JO, Adhikari NK, Beyene J. Ratio of geometric means to analyze continuous outcomes in meta-analysis: comparison to mean differences and ratio of arithmetic means using empiric data and simulation. Stat Med. 2012;31(17):1857-1886

17. Friedrich JO, Adhikari NK, Beyene J. Ratio of means for analyzing continuous outcomes in meta-analysis performed as well as mean difference methods. J Clin Epidemiol. 2011;64(5):556-564.

18. Frazzetta M, Furgiuele G, Raimondo D, et al. [Ultrasonic dissector for total thyroidectomy: results of prospective randomized study] Tiroidectomia totale con impiego del dissettore ad ultrasuoni: risultati di uno studio prospettico randomizzato. G Chir. 2005;26(8-9):295-301. Italian [with English abstract].

19. Hallgrimsson P, Lovén L, Westerdahl J, Bergenfelz A. Use of the harmonic scalpel versus conventional haemostatic techniques in patients with Grave disease undergoing total thyroidectomy: a prospective randomised controlled trial. Langenbecks Arch Surg. 2008;393(5):675-680.

20. Hubner M, Demartines N, Muller S, Dindo D, Clavien PA, Hahnloser D. Prospective randomized study of monopolar scissors, bipolar vessel sealer and ultrasonic shears in laparoscopic colorectal surgery. $\mathrm{Br} J$ Surg. 2008;95(9):1098-1104.

21. Inoue $\mathrm{K}$, Nakane $\mathrm{Y}$, Michiura $\mathrm{T}$, et al. Ultrasonic scalpel for gastric cancer surgery: a prospective randomized study. J Gastrointest Surg. 2012;16(10):1840-1846.

22. Konturek A, Barczyński M, Stopa M, Nowak W. Total thyroidectomy for non-toxic multinodular goiter with versus without the use of harmonic FOCUS dissecting shears - a prospective randomized study. Wideochir Inne Tech Maloinwazyjne. 2012;7(4):268-274.

23. Kowalski LP, Sanabria A, Vartanian JG, et al. Total thyroidectomy with ultrasonic scalpel: a multicenter, randomized controlled trial. Head Neck. 2012;34(6):805-812.

24. Laycock WS, Trus TL, Hunter JG. New technology for the division of short gastric vessels during laparoscopic Nissen fundoplication. A prospective randomized trial. Surg Endosc. 1996;10(1):71-73.

25. Lombardi CP, Raffaelli M, Cicchetti A, et al. The use of "harmonic scalpel" versus "knot tying" for conventional "open" thyroidectomy: results of a prospective randomized study. Langenbecks Arch Surg. 2008;393(5):627-631.

26. Pons Y, Gauthier J, Ukkola-Pons E, et al. Comparison of LigaSure vessel sealing system, harmonic scalpel, and conventional hemostasis in tota thyroidectomy. Otolaryngol Head Neck Surg. 2009;141(4):496-501.

27. Swanstrom LL, Pennings JL. Laparoscopic control of short gastric vessels. J Am Coll Surg. 1995;181(4):347-351.

28. Targarona EM, Balague C, Marin J, et al. Energy sources for laparoscopic colectomy: a prospective randomized comparison of conventional electrosurgery, bipolar computer-controlled electrosurgery and ultrasonic dissection. Operative outcome and costs analysis. Surg Innov. 2005;12(4):339-344.
29. Tempé F, Jänes A, Cengiz Y. Cost analysis comparing ultrasonic fundus-first and conventional laparoscopic cholecystectomy using electrocautery. Surg Endosc. 2013;27(8):2856-2859.

30. Uzunoglu FG, Stehr A, Fink JA, et al. Ultrasonic dissection versus conventional dissection techniques in pancreatic surgery: a randomized multicentre study. Ann Surg. 2012;256(5):675-679; discussion 679-680.

31. Fingar KR, Stocks C, Weiss AJ, Steiner CA. Most Frequent Operating Room Procedures Performed in U.S. Hospitals, 2003-2012. Available from: http://www.hcup-us.ahrq.gov/reports/statbriefs/sb186-OperatingRoom-Procedures-United-States-2012.pdf.

32. Doheny K. For best thyroid surgery results, pick doctor with 25-plus cases a year [updated November 05, 2016]. Available from: https:// www.endocrineweb.com/news/thyroid-diseases/20364-best-thyroidsurgery-results-pick-doctor-25-plus-cases-year. Accessed October 02, 2017.

33. Chatterjee A, Payette MJ, Demas CP, Finlayson SR. Opportunity cost: a systematic application to surgery. Surgery. 2009;146(1):18-22.

34. Grochola LF, Vonlanthen R. Surgical energy devices or devices for hemostasis. In: Clavien PA, Sarr MG, Fong Y, Miyazaki M, editors. Atlas of Upper Gastrointestinal and Hepato-Pancreato-Biliary Surgery. Berlin:Springer. 2016:37-44.

35. Cheng H, Soleas I, Ferko NC, Clymer JW, Amaral JF. A systematic review and meta-analysis of Harmonic Focus in thyroidectomy compared to conventional techniques. Thyroid Res. 2015;8:15.

36. Catena F, Di Saverio S, Ansaloni L, et al. The HAC trial (harmonic for acute cholecystitis): a randomized, double-blind, controlled trial comparing the use of harmonic scalpel to monopolar diathermy for laparoscopic cholecystectomy in cases of acute cholecystitis. World $J$ Emerg Surg. 2014;9(1):53.

37. Polacco MA, Pintea AM, Gosselin BJ, Paydarfar JA. Parotidectomy using the Harmonic scalpel: ten years of experience at a rural academic health center. Head Face Med. 2017;13(1):8.

38. Shippert RD. A study of time-dependent operating room fees and how to save $\$ 100000$ by using time-saving products. Am J Cosmet Surg. 2005;22(1):25-34.

39. Contin P, Gooßen K, Grummich K, et al. ENERgized vessel sealing systems versus CONventional hemostasis techniques in thyroid surgery--the ENERCON systematic review and network meta-analysis. Langenbecks Arch Surg. 2013;398(8):1039-1056.

40. Bulus H, Basar O, Tas A, et al. Evaluation of three instruments for laparoscopic cholecystectomy: harmonic scalpel, bipolar vessel sealer, and conventional technique. Minerva Chir. 2013;68(6):537-542.

41. Underwood RA, Dunnegan DL, Soper NJ. Prospective, randomized trial of bipolar electrosurgery vs ultrasonic coagulation for division of short gastric vessels during laparoscopic Nissen fundoplication. Surg Endosc. 1999;13(8):763-768.

42. Burdette TE, Kerrigan CL, Homa K. Harmonic scalpel versus electrocautery in breast reduction surgery: a randomized controlled trial. Plast Reconstr Surg. 2011;128(4):243e-249e.

43. Holub Z, Voracek J, Jun LK, Lukac J. Laparoscopic hysterectomy: randomized study of harmonic scalpel and electrosurgery. $J$ Gynecol Surg. 2000;16(1):33-39.

44. Holub Z, Jabor A, Kliment L, Voracek J, Lukac J, Barany B. Laparoscopic staging of endometrial cancer using laparosonic instruments: comparison with electrosurgery. Eur J Obstet Gynecol Reprod Biol. 2001;100(1):81-86.

45. Fitz-Gerald AL, Tan J, Chan KW, et al. Comparison of ultrasonic shears and traditional suture ligature for vaginal hysterectomy: randomized controlled trial. J Minim Invasive Gynecol. 2013;20(6):853-857.

46. Kawabata R, Takiguchi S, Kimura Y, et al. A randomized phase II study of the clinical effects of ultrasonically activated coagulating shears (Harmonic scalpel) in open gastrectomy for gastric cancer. Surg Today. 2016;46(5):561-568.

47. Ortega J, Sala C, Flor B, Lledo S. Efficacy and cost-effectiveness of the UltraCision harmonic scalpel in thyroid surgery: an analysis of 200 cases in a randomized trial. J Laparoendosc Adv Surg Tech A . 2004;14(1):9-12. 
48. Balshem H, Stevens A, Ansari M, et al. Finding Grey Literature Evidence and Assessing for Outcome and Analysis Reporting Biases When Comparing Medical Interventions: AHRQ and the Effective Health Care Program. Methods Guide for Comparative Effectiveness Reviews. (Prepared by the Oregon Health and Science University and the University of Ottawa Evidence-based Practice Centers under Contract Nos. 290-2007-10057-I and 290-2007-10059-I.) AHRQ Publication No. 13(14)-EHC096-EF. Rockville, MD: Agency for Healthcare Research and Quality. November 2013.
49. Tam VC, Hotte SJ. Consistency of phase III clinical trial abstracts presented at an annual meeting of the American Society of Clinical Oncology compared with their subsequent full-text publications. J Clin Oncol. 2008;26(13):2205-2211.

\section{Publish your work in this journal}

ClinicoEconomics and Outcomes Research is an international, peerreviewed open-access journal focusing on health technology assessment, pharmacoeconomics and outcomes research in the areas of diagnosis, medical devices, and clinical, surgical and pharmacological intervention. The economic impact of health policy and health systems organization also constitute important areas of coverage. The manuscript management system is completely online and includes a very quick and fair peer-review system, which is all easy to use. Visit http://www.dovepress.com/testimonials.php to read real quotes from published authors. 